\title{
Gap-Plasmon-Enhanced Nanofocusing Near-Field Microscopy
}

\author{
Simon F. Becker, ${ }^{\star \dagger}$ Martin Esmann, ${ }^{\dagger}$ KyungWan Yoo, ${ }^{\dagger, \ddagger}$ Petra Gross, ${ }^{\dagger}$ Ralf Vogelgesang, ${ }^{\dagger}$ \\ NamKyoo Park, ${ }^{\ddagger}$ and Christoph Lienau ${ }^{\dagger}$ \\ ${ }^{\dagger}$ Institut für Physik and Center of Interface Science, Carl von Ossietzky Universität, D-26111 Oldenburg, Germany \\ ${ }^{\ddagger}$ Photonic Systems Laboratory, School of EECS, Seoul National University, Seoul 151-744, South Korea
}

KEYWORDS: nanofocusing, near-field microscopy, gap plasmon, nanospectroscopy

\section{Discussion of Point Dipole Models}

Frequently, far-field signals from apertureless scanning near-field optical microscopes (SNOMs) are discussed in the framework of point dipole models, ${ }^{1-2}$ and the immediate question arises, whether the physics behind the main features of our approach curves might be captured already by such a comparatively simple model. Therefore, we assess here the applicability of point dipole models.

The central assumption of all point dipole models is that the probe tip may be represented by a single point-like dipole, situated at the center of a sphere whose radius is given by the local effective radius of curvature $R$ at the apex. Conventionally, an external field excites the dipole both directly and indirectly via reflection at the sample surface. In the present context, the adiabatically illuminated tip dipole itself acts as the sole source of excitation. The dipole is oriented normal to the sample surface. In the quasi-static limit, the near-field optical interaction over a tip-sample distance $d$ (surface-tosurface) results in an effective polarizability of the system given as

$$
\alpha_{e f f}=\left(\alpha^{-1}-\frac{1}{16 \pi \varepsilon_{0}} \frac{\beta}{(R+d)^{3}}\right)^{-1}
$$

Here, $\varepsilon_{0}$ is the dielectric constant of the medium between tip and sample, whose respective dielectric constants are $\varepsilon_{t}$ and $\varepsilon_{s}$, and $\beta=\left(\varepsilon_{s}-\varepsilon_{0}\right) /\left(\varepsilon_{s}+\varepsilon_{0}\right)$ is the reflection coefficient of the sample surface. The polarizability of the isolated spherical tip is $\alpha=4 \pi R^{3} \varepsilon_{o}\left(\varepsilon_{t}-\varepsilon_{0}\right) /\left(\varepsilon_{t}+2 \varepsilon_{0}\right)$. In the dipole model the observable scattered intensity is proportional to the absolute square of the effective polarizability. In the present case, we have additional factors due to the evanescent decay $\propto \exp \left(-d / L_{D}\right)$ of the observed leaky modes above the sample surface and the transmissivity of the sample. Here, $L_{D}$ is the decay length on intensity level. Algebraically, the effective polarizability has the form of a resonance denominator. It seems conceivable at first glance that the maximum observed for distances $d \approx 110 \mathrm{~nm}$ might be due to the denominator nearly vanishing, which would lead to a so-called configurational resonance. ${ }^{3}$ A careful evaluation of the present case, however, reveals that this is not realizable for $d \geq 0$. Both the sphere's polarizability $\alpha$ and the surface reflectivity $\beta$ are too small. The tip-sample system remains in the weak coupling regime, since $16 \pi \varepsilon_{0}(R+d)^{3}>\alpha \beta$. Only if the product $\alpha \beta$ would be bigger by a factor of $\sim 3.3$ or more, strong coupling could set in and the Taylor expansion $\alpha_{\text {eff }} \approx \alpha[1+$ $\left.\alpha \beta /\left(16 \pi \varepsilon_{0}\right) \cdot(R+d)^{-3}+\cdots\right]$ would fail. A noticeable increase of scattered radiation is predicted only for $d \leq R$, i.e., within the last few nanometers of an approach curve. This behavior closely resembles the sharp intensity maximum observed in near-contact in Figure 2(d). The main maximum near $110 \mathrm{~nm}$, however, is not explained.

A more realistic version of the point dipole model includes all retardation effects. ${ }^{3-6}$ Using the Green function formalism with the Weyl identity, the coupling problem between the point like spherical tip and a multilayer sample system is solved self-consistently, resulting in an effective polarizability tensor

$$
\overleftrightarrow{\alpha}_{e f f}=\left(\overleftrightarrow{\alpha}^{-1}-\overleftrightarrow{W}\right)^{-1}
$$

where $W_{i, j}=i\left(8 \pi^{2} \varepsilon_{0}\right)^{-1} \iint d^{2} k_{\|} r_{i l}\left(\vec{k}_{\|}\right)\left(k_{0}^{2} \delta_{j, l}-k_{j} k_{l}\right) e^{i 2 k_{z}(R+d)} / k_{z}$ describes the self-interaction of the dipole due to reflection of its emitted field at the sample surface. The reflection tensor $r_{i l}\left(\vec{k}_{\|}\right)$for the multilayer sample is obtained from a transfer matrix formalism. The quasi-static dipole model represents the limiting case of this dynamic model for small tipsample distances and fields evaluated in close proximity to the tip dipole. Therefore, we find essentially the same behavior 
for $d<R$ as in the quasi-static case. For larger distances, the magnitude of $W$ diminishes rapidly relative to that of the inverse polarizability $\alpha^{-1}$ of the isolated sphere dipole - again in close resemblance to the quasi-static case. Consequently, all versions of the point dipole model fail to explain the maximum near $d \approx 110 \mathrm{~nm}$.

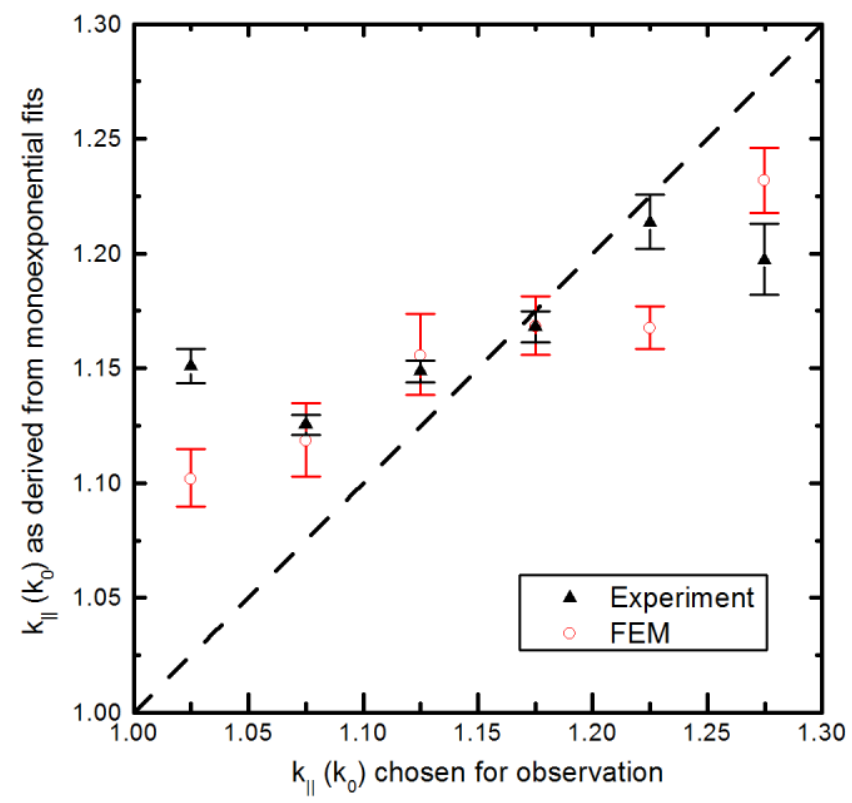

Figure S1. Exponential decay of the approach curves $I\left(d, k_{\|}\right)$for large tip-sample distances $d=200 \ldots 500 \mathrm{~nm}$. For different in-plane wavevector components $k_{\|}$chosen for observation, an exponential decay $I \propto \exp \left(-d / L_{D}\right)$ was least-squares fitted to experimental (black triangles) and simulated data (red circles). The corresponding wavevector component was derived as $k_{\|}=\sqrt{k_{0}^{2}+\left(2 L_{D}\right)^{-2}}$. The vertical error bars represent the $95 \%$ confidence intervals of the fits.

Another discrepancy of the dipole models with experimental observation is found in the exponential decay of the recorded intensity for distances $d>200 \mathrm{~nm}$. At such distances the point dipole is only weakly coupled to the surface. Hence, the observed intensity for evanescent field components $\left(k_{\|}>k_{0}\right)$ should exhibit a simple exponential decay, $I(d) \propto$ $\exp \left(-d / L_{D}\right)$, with the decay constant $L_{D}$ on the intensity level depending on the in-plane wavevector component as $k_{\|}^{2}=k_{0}^{2}+\left(2 L_{D}\right)^{-2}$. In Figure $\mathrm{S}_{1}$ this expectation is indicated by the dashed black line. In stark contrast, we find that both experiment and finite element method (FEM) simulation exhibit a decay constant that depends very little on the in-plane wavevector component, as shown in Figure S1.

\section{Calibration of Tip-sample Distance}

Our setup employs an atomic force microscope (AFM) oscillating the AFM probe normal to the sample surface. The probe is attached to a tuning fork with a stiffness large enough to allow operation in the attractive force or non-contact regime, avoiding any snap-in events. The free probe oscillation amplitude at large distances is well below $1 \mathrm{~nm}$. An experimental calibration of the tip-sample distance involves physical contact between probe and sample in the repulsive force regime. This generally deforms or destroys the sharp apex of our gold taper probes, which must be avoided in SNOM experiments. Hence, we estimate the tip-sample distance in SNOM experiments by comparison with independent tests in which we actually did drive probe tips of similar geometry into the sample in a controlled manner.

A corresponding typical approach curve is shown in Figure S2. It shows the tuning fork tapping amplitude normal to the surface as a function of sample position measured with an independent capacitive sensor (black squares). The data were fitted to a model from Karrai \& Tiemann ${ }^{7}$ (red solid line). For small distances we observe saturation of the decreasing signal, which we interpret as direct contact between tip and sample, corresponding to vanishing gap size. The signal change from "free" to "in contact" happens over a distance of $2.1 \pm 0.1 \mathrm{~nm}$ (using $90 \%$ and $10 \%$ criteria). In our experiments presented in the manuscript, the approach curves were stopped, when the amplitude signal fell below a threshold of $85 \%$ of the absolute signal. In Figure $\mathrm{S}_{2}$ this corresponds to an oscillation amplitude of $0.57 \mathrm{~nm}_{\mathrm{rms}}=0.81 \mathrm{~nm}_{\text {peak }}$, and this damped amplitude is encountered at a distance of $1.8 \mathrm{~nm}$. That is, the average tip sample distance at the approach curve stopping condition is $1.8 \pm 0.1 \mathrm{~nm}$ in Figure S2, and the tip is oscillating with an amplitude of about $0.8 \mathrm{~nm}$. These values are typical for our measurements. 


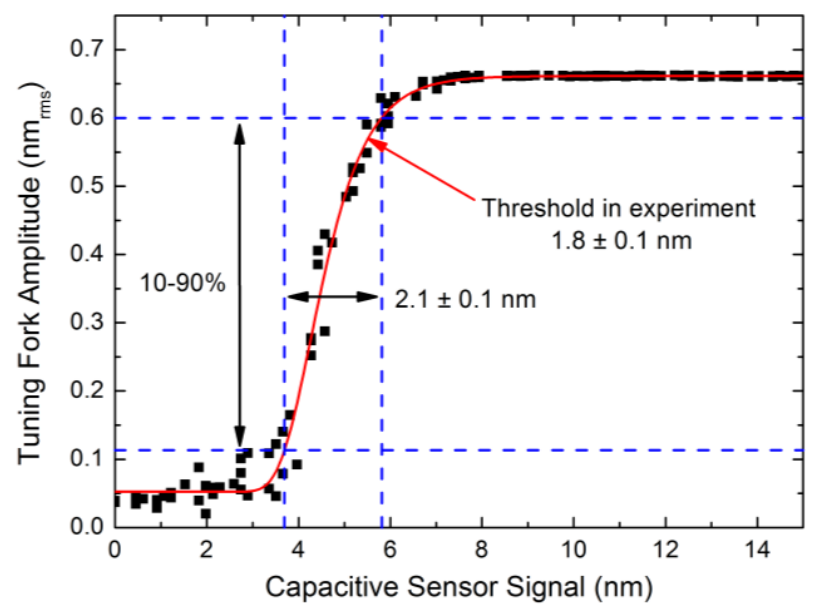

Figure S2. Typical shape of the tuning fork tapping amplitude as a function of an independent capacitive sensor signal with arbitrary zero measuring the sample position when driving a tip into the sample (black squares) along with a corresponding fit according to Karrai \& Tiemann ${ }^{7}$ (red solid lines).

\section{Influence of Relative Tilt}

In this section we discuss a possible reason for the rotational anisotropy seen in the angle-resolved approach curves in Figure 2. The FEM simulations presented in the main text are performed for a rotationally symmetric tip-sample geometry and hence do not account for these deviations. Here, we consider a slight misalignment between the surface normal of the sample and the symmetry axis of the taper of about $10^{\circ}$. We performed a two-dimensional FEM simulation to investigate the effect of this misalignment on the angle-resolved approach curves.

The simulation was carried out using the finite element method (COMSOL Multiphysics) in $2 \mathrm{D}$ mode (x-z plane) with full vectorial field components. The size of the simulation domain containing gold taper and gold-coated glass substrate was $7 \mu \mathrm{m}$ (x direction) by $5.5 \mu \mathrm{m}$ (z direction), including $800 \mathrm{~nm}$ wide perfectly matched layers. The apex radius and opening angle of the taper were set to $10 \mathrm{~nm}$ and $25^{\circ}$, respectively. The thickness of the film was set to $30 \mathrm{~nm}$ and the film was tilted by $10^{\circ}$ from the $\mathrm{z}=$ constant plane in counter-clockwise direction. The relative permittivity for gold $\varepsilon_{A u}=$ $-24.747+1.8834 i$ at $\lambda_{0}=800 \mathrm{~nm}$ was taken from a fit to literature data. ${ }^{8}$ The value for glass was set to $\varepsilon_{\text {glass }}=2.28$ $\left(\mathrm{BK}_{7}\right)$. By using port boundary conditions at the upper end of the simulation domain, we can excite a taper eigenmode of the taper and also absorb waves which propagate into this boundary. To keep the excitation power and also modal shape the same for all tip-sample spacings $d$, the position of the taper was fixed while changing the position of the gold-coated glass substrate. We define the tip-sample spacing as the distance between the taper apex and the film surface in vertical direction rather than as the shortest distance between taper and film. The maximum mesh size in air and glass was set to $\lambda_{\text {air }} / 20$ and $\lambda_{\text {glass }} / 20$, respectively, except for the vicinity of the apex where the maximum mesh size was set to $0.1 \mathrm{~nm}$. All calculations were verified by convergence tests. The field distribution was extracted in a plane inside the glass halfspace and $1 \mathrm{~nm}$ away from the gold-glass interface and subsequently converted to $1 \mathrm{D} k$-space data using the Fourier transform. To obtain approach curves comparable to those discussed in the main text $|\boldsymbol{E}|^{2}$ was integrated in the region $1<\left|k_{\|}\right|<1.3$ for $+k_{\|}$and $-k_{\|}$directions separately. 

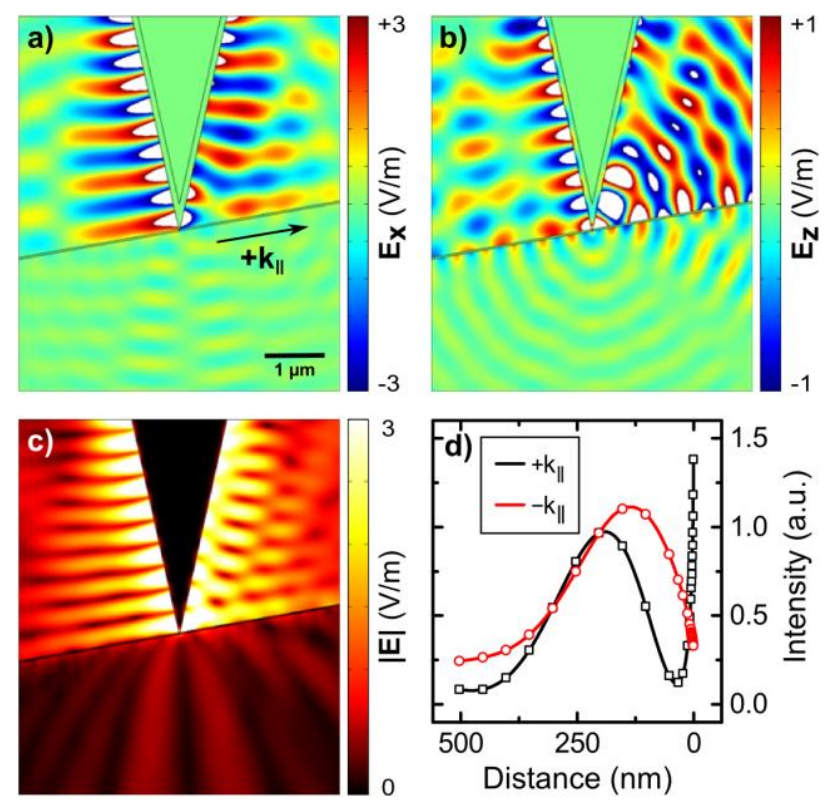

Figure S3. (a-c) FEM calculations of the field distribution around a metallic taper above a $30 \mathrm{~nm}$ thick gold film on top of a glass substrate tilted by $10^{\circ}$ for a vertical tip-sample distance of $0.5 \mathrm{~nm}$. Panels (a-c) show patterns of $E_{x}, E_{z}$, and $|\boldsymbol{E}|$, respectively. (d) Approach curves extracted from field distributions as shown in panels (a-c) evaluated for different tip-sample distances. The data are calculated by integrating $|\boldsymbol{E}|^{2}$ in the region $1<\left|k_{\|}\right|<1.3$. The black and red curves show data for $+k_{\|}$and $-k_{\|}$directions, respectively.

Figure $S_{3}(\mathrm{a}-\mathrm{c})$ shows the resulting field distribution around the tip-sample system. Panel (b) reveals that when the sample is tilted out of the plane normal to the taper axis by $10^{\circ}$, a highly asymmetric signal is observed at the in-plane wavevector components corresponding to $\mathrm{k}_{\|} \approx \pm 1.027 \mathrm{k}_{0}$. Whereas on the right side $\left(\mathrm{k}_{\|} \approx+1.027 \mathrm{k}_{0}\right)$ leakage radiation is evidently being emitted, on the left side $\left(\mathrm{k}_{\|} \approx-1.027 \mathrm{k}_{0}\right)$, the signal is much reduced. In panel $(\mathrm{d})$ the data are analyzed as a function of tip-sample distance as described above. The corresponding approach curves exhibit a clear peak over the last few nanometers for $\mathrm{k}_{\|} \approx+1.027 \mathrm{k}_{0}$ and a nearly complete loss of this gap plasmon signature for $\mathrm{k}_{\|} \approx-1.027 \mathrm{k}_{0}-$ in analogy with our experimental findings discussed in the manuscript.

\section{AUTHOR INFORMATION}

\section{Corresponding Author}

*simon.f.becker@uni-oldenburg.de

\section{Author Contributions}

S.F.B. and M.E. did the experiments. K.W.Y. performed the FEM simulations. The manuscript was written through contributions of all authors. All authors have given approval to the final version of the manuscript.

\section{ABBREVIATIONS}

AFM, atomic force microscopy; FEM, finite element method; SNOM, scanning near-field optical microscopy.

\section{REFERENCES}

1. Knoll, B.; Keilmann, F., Enhanced dielectric contrast in scattering-type scanning near-field optical microscopy. Opt Commun 2000, 182, 321-328.

2. $\quad$ Koglin, J.; Fischer, U. C.; Fuchs, H., Material contrast in scanning near-field optical microscopy at 1-10 nm resolution. Phys Rev $B$ 1997, 55, 7977-7984.

3. Keller, O.; Xiao, M. F.; Bozhevolnyi, S., Configurational Resonances in Optical near-Field Microscopy - a Rigorous PointDipole Approach. Surf Sci 1993, 280, 217-230.

4. Xiao, M.; Bozhevolnyi, S.; Keller, O., Numerical study of configurational resonances in near-field optical microscopy with a mesoscopic metallic probe. Appl Phys a-Mater 1996, 62, 115-121.

5. Xiao, M. F., Evanescent field coupling of dipole to a surface: Configurational resonance at long distances. Chem Phys Lett 1996, 258, 363-368.

6. Hecht, B.; Novotny, L., Principles of Nano-Optics. 2nd ed.; Cambridge University Press: Cambridge, 2012.

7. $\quad$ Karrai, K.; Tiemann, I., Interfacial shear force microscopy. Phys Rev B 2000, 62, 13174-13181.

8. Johnson, P. B.; Christy, R. W., Optical Constants of Noble Metals. Phys Rev B 1972, 6, 4370-4379. 